\title{
CLINICAL ASSESSMENT OF THE NEWER ANTIBIOTICS
}

By Prof. Clifford Wilson

The London Hospital

In recent years new and potent antibiotics have been introduced with a disturbing frequency and developments of industrial and research methods will doubtless produce a continuing series of these drugs. The practitioner is at once stimulated by the prolific and optimistic claims of the manufacturers and inhibited by restrictions of supply. The discriminating use of these new and powerful drugs and the selection of the most appropriate antibiotic for the individual patient are matters of the greatest importance. This article is mainly concerned with the clinical assessment of aureomycin, chloramphenicol and terramycin in the treatment of infectious disease.

It has been said that every discovery raises a new problem. The discovery in this country and elsewhere of a succession of antibiotics has posed a whole series of problems during the past five years and it is interesting to see how these problems have been dealt with by those concerned. The bacteria have faced the biggest problem - that of survivaland their solution has been the development of drug resistance. Next comes the scientist, who has been set the recurring problem of discovering new antibiotics which will deal with the resistant bacteria and which have a wide range of action devoid of toxic side effects; in short as the bacterial population develops new defences, he has to keep one jump ahead with a new offensive weapon. Following closely comes the bacteriologist, to whom the fundamental problem is the mechanism of action. What is the effect of antibiotic therapy on the natural development of immunity? Does it attack the bacteria through some specific metabolic process? On the more practical side, the bacteriologist is faced with the estimation of antibiotic levels in body fluids and the time-consuming routine of sensitivity testing. Next in sequence comes the manufacturer; the processes of fermentation and purification on a commercial scale are costly, and for him the problem is to recoup his capital outlay before a newer and better anti- $\dot{\omega}$ biotic appears. This means a high selling price $N$ during the first few years which explains why treatment with the new antibiotics is some ten times as expensive as with penicillin. This policy 은 in turn poses a financial problem for the consumer, and in this country those who are responsible for distribution of these drugs under the National Health Service are particularly affected, for the $\stackrel{\text { क }}{+}$ general distribution of one of the néwer anti $\vec{\theta}$ biotics adds a considerable burden to the cost of on the service.

The most important problem of all, and the one which is most urgent for us as doctors and for the patient is 'which antibiotic?' Unlike the previous problems this one unfortunately cannot be satisfactorily answered in simple terms by general rules. The right answer is always particular to the individual patient and this becomes even more essential when we consider the use of the newer antibiotics aureomycin, chloramphenicol and terramycin. In arriving at the proper solution there is obviously a conflict of interest between those who produce and those who prescribe antibiotics. The manufacturer is obviously desirous of producing a non-toxic, wide range antibiotic which will find the most general application. Indeed a suggestion was at one time made that the use of aureomycin in pneumonia made exact diagnosis no longer necessary. This is a dangerous doctrine, for most of us have encountered patients suffering from pulmonary tuberculosis or neoplasm of the lung, whose condition has steadily deteriorated during treatment with antibiotics before diagnosis had been properly established. Thus the search for an all-purposes antibiotic is a mistaken one and in the treatment of the sick patient bears the same stigma of unreality as the panaceas and elixirs of the mediaeval apothecary.

In dealing with any perplexing problem, the historical approach to the subject is often the short 
cut to a clear understanding, and I believe this is the best method of assessing the newer antibiotics. The discovery of penicillin has provided us with a relatively inexpensive, non-toxic anti-bacterial agent. Its disadvantages are that for prompt and effective action it has to be given by injection and it has little effect on a wide range of infections, the most important of which are tuberculosis, virus infections, many gram-negative bacillary infections of the urinary tract and common epidemic diseases such as enteric fever, brucellosis, typhus and whooping cough. The next antibiotic, streptomycin, provided the first effective weapon against tuberculosis and it had considerable influence on resistant gram-negative bacillary infections, e.g. in the urinary tract. Like penicillin it had to be given by injection; unlike penicillin it produced serious side-effects, especially vertigo and nerve deafness. Dihydro-streptomycin has similar and perhaps graver toxic effects.

The search for antibiotics with an even wider antibacterial range continued therefore, and aureomycin, chloramphenicol and terramycin appeared in quick succession and have come into general use during the last two years. These three antibiotics have more similarities than differences, and this in itself makes more difficult the problem of choice. Chloramphenicol is unique in being the first antibiotic to be synthesised on a commercial scale. It is the only one of the three at present generally available on prescription in this country, the distribution of aureomycin and terramycin, which have to be imported, being restricted to hospitals. The three antibiotics have also almost identical toxic effects, which produce gastrointestinal symptoms such as nausea, vomiting, stomatitis and proctitis due to the occurrence of resistant mycotic infections; these infections, although not serious in themselves are unpleasant and may prohibit continued therapy in chronic conditions such as brucellosis. Chloramphenicol also depresses haemopoiesis and its continued administration may lead to granulopaenia and anaemia. These side effects have to be taken into account in comparing the new antibiotics with other forms of treatment. Another factor which influences our choice of antibiotic is the development of bacterial resistance. Many strains of staphylococci are resistant to penicillin owing to penicillinase production. Streptomycin, which may have a potent anti-bacterial action in penicillin resistant infections of the urinary tract, becomes ineffective in many cases owing to the development of bacterial resistance or the emergence of organisms whose growth is actually promoted by streptomycin. The development of resistance to the newer antibiotics is uncommon and of moderate degree. It has been seen chiefly in staphylococci which may become resistant to both aureomycin and terramycin; resistance to chloramphenicol is less $\mathbb{\Phi}$ common. As with other antibiotics, the new ${ }_{\subset}$ drugs by early suppression of the infection may prevent the development of active immunity. It has been found for instance that successful treat-o ment of typhoid may be followed by re-develop-흘 ment of the disease due to infection with a different $\frac{\bar{m}}{\frac{5}{}}$ phage type.

\section{Specific Indications}

The specific indications for the use of the new. antibiotics can be derived only from experience of $\vec{\omega}$ therapeutic trials in various infections. The first $\stackrel{S}{\circ}$ guide is, of course, in vitro sensitivity. Most ofo the published work on the three new antibiotics 3 . comes from the United States. In this countryi therapeutic trials have been sponsored by the Medical Research Council and a special antibiotic N committee has been working on these problems for the past three years. The results of some of these ${ }^{v}$ trials are in process of publication.

At the outset I must mention certain important problems which arise in the conduct of therapeutic trials. From the point of view of antibiotic therapy infections fall into two groups. First we have the group of infections for whighe there is no known effective anti-bacterial therasy and on which the new drug has an obvious specifici effect. The value of a new drug is therefore be yond doubt in such cases providing there are no serious side-effects. If, in addition, the disease carries a high mortality, the results of treatment $\overline{0}$ are easy to assess without elaborate control $\stackrel{\varrho}{\rightleftarrows}$ observations. Such, for example, was the case in음 the treatment of subacute bacterial endocarditis with penicillin, and of tuberculous meningitis with streptomycin.

On the other hand there is a large variety of $\frac{}{3}$ infectious diseases which have a variable natural ${ }_{-}^{-}$. history and which often resolve spontaneously? owing to the normal defensive reactions of the? body. In these it is difficult to assess the value of a new antibiotic and even more difficult to com-o pare two similar antibiotics without a carefully controlled therapeutic trial. The introduction of such controlled trials in this country has been one $\mathrm{N}$. of the major contributions to antibiotic therapy. Or The procedure is well illustrated by the investiga- $N$ tion of streptomycin therapy of pulmonary N tuberculosis sponsored by the Medical Research $\omega$ Council. Essential features of such trials are, cooperation between different groups of observers $\frac{0}{\mathbb{}}$ in order to study the response in a large series of $\stackrel{0}{\rightarrow}$ patients, the division of cases into treated and 0 control groups in such a way that the groups are $\bar{O}^{\circ}$ comparable, the planning of treatment if possible $\vec{\otimes}$ in such a manner that neither patients nor ob- $-\frac{\mathbb{d}}{\mathbb{Q}}$ 
servers are aware whether the antibiotic or the control substance is being administered to any particular individual and, finally, the statistical examination of results. Such therapeutic trials must, therefore, be worked out in full detail in advance and the final assessment of results must be made by independent observers. It is the absence of controlled observations which often makes it impossible to assess accurately the claims which are so graphically and persuasively presented in the trade advertisements.

In the clinical evaluation of aureomycin, chloramphenicol and terramycin there are certain diseases which fall into the first group of infections described above; these diseases may therefore be accepted as definite indications for the use of the drugs.

First we have the rickettsial infections. The therapeutic trial of chloramphenicol in typhus fever is one of the classic contributions to antibiotic therapy. Subsequent work has shown that all three antibiotics are effective against typhus; $\mathrm{Q}$ fever is the only rickettsial infection likely to be encountered in this country. The therapeutic value of chloramphenicol in typhoid fever was accidentally discovered during the investigation of typhus. As previously mentioned early treatment interferes with the development of immunity and relapses may occur when administration is discontinued, hence chloramphenicol should be given in typhoid for at least 18 days. This may be followed by vaccine therapy in an attempt to stimulate immunity. Perforation and haemorrhage may follow subsidence of fever so that a careful watch for complications must be maintained for some time.

Certain virus infections, particularly of the psittacosis-lymphogranuloma group respond to these three antibiotics; virus pneumonia also appears to respond satisfactorily.

All three antibiotics are effective against brucellosis, but the infection is difficult to eradicate; relapses are common and the appearance of sideeffects may interfere with continued therapy. It was indeed as a result of long-continued therapy of chronic brucellosis that mycotic infections of the gastro-intestinal tract were first recognized as an undesirable complication of aureomycin and chloramphenicol therapy-although both drugs had been widely used for some 18 months. Most of the published work on brucellosis reports the use of aureomycin; chloramphenicol is equally effective but continued therapy may have a depressing effect on the bone marrow. There is less information on terramycin therapy of brucellosis but it has been claimed that stomatitis and proctitis occur less often.

Penicillin-resistant staphylococcal infection may also be included as a definite indication for these antibiotics. In vitro the organism is particularly 3 sensitive to aureomycin, but good clinical results $\stackrel{\unrhd}{\varrho}$ have been reported with chloramphenicol. Thus $C$ in staphylococcal bacteraemia, staphylococcal $\Rightarrow$ endocarditis, meningitis and local infections such $\stackrel{5}{?}$ as otitis and sinusitis, any one of these antibiotics provides effective primary treatment.

Our real difficulty arises when we try to assess $\frac{0}{2}$ the value of aureomycin, chloramphenicol and $\varnothing$ terramycin in the second group of diseases where the natural history is variable and recovery is the $\overrightarrow{0}$ rule. This group includes a wide range of infections such as pneumonia, urinary tract in- $\vec{\omega}$ fections, whooping cough, glandular fever, ab- $\frac{\rho}{\circ}$ dominal infections including peritonitis, and numerous skin eruptions; wide claims have been made, on alleged evidence or by implication, and the evaluation of these claims is difficult since the therapeutic trials on which they are based have rarely been properly controlled.

\section{Respiratory Infections}

Pneumonia. Numerous investigators have reported the effective control of pneumococcal pneumonia by the newer antibiotics and all three appear to be equally effective. It is doubtful however, whether they should replace penicillin as standard treatment. In a controlled trial the Pneumonia Working Party of the Medical Researc Council (Brit. med. F., ii, 1361 ) treated a series of 267 cases in three groups, comparing the effect of chloramphenicol, aureomycin and standard treatment with penicillin or sulphonamides. No attempt was made to select cases according to anatomical or bacteriological diagnosis. The results of this trial indicate that the penicillin is at least as effective as aureomycin and chloramphenicol and is to be preferred as primary treatment when the side effects of the newer antibiotics are taken into account. It must be recognized, however, that pneumonia is occasionally caused by penicillin resistant organisms, e.g. Friedlander's bacillus or $H$. influenzae, hence bacterial diagnosis should preferably be made in all patients and certainly in resistant cases so that the most effective antibiotic can be given without delay.

Whooping cough. A few reports have been published both in this country and the U.S.A. claiming beneficial results from all three antibiotics. It is probable that the course of the disease is appreciably shortened only if treatment is started during the first week of the disease. Both 0 chloramphenicol and terramycin can be administered in a pleasantly flavoured elixir suitable for small children.

Acute tracheo-laryngo-bronchitis in infants. The bacterial cause of this disease is obscure but it 
carries a generally high mortality. A few cases have been treated with chloramphenicol with apparent dramatic benefit, hence the use of the drug in this condition is justifiable.

Bronchiectasis. Certain claims have been made for all three antibiotics in this condition. In the acute phases or during pneumonic exacerbations penicillin-resistant organisms often respond to aureomycin, chloramphenicol or terramycin, but there is so far no definite evidence that continued therapy is beneficial. $H$. influenzae is commonly found in such cases after penicillin treatment, and it may be eradicated by aureomycin and chloramphenicol. After treatment, however, the organism may return without the re-appearance of clinical symptoms.

\section{Gastro-Intestinal Tract}

Apart from typhoid fever there appear to be no clear specific indications for the newer antibiotics. It is important to note that in the faecal carrier state $S$. typhi cannot be eradicated by chloramphenicol. Bacillary dysentery is best treated with sulphonamides. Amoebic dysentery responds well to both aureomycin and terramycin, but the final assessment in these conditions requires more extended trials. Tentative claims have been made for the new antibiotics in infantile gastro-enteritis. Favourable results with chloramphenicol have been reported but it is doubtful whether these are significantly different from the effect of treatment with sulphonamides. In any case general supportive measures, especially rehydration, are essential.

A number of reports have appeared on the use of the new antibiotics in suppurative peritonitis. Although penicillin is acknowledged to be of great value and has produced an undoubted improvement in the prognosis of abdominal sepsis, nevertheless penicillin-resistant organisms are usually present and the new antibiotics might be expected to be more effective. When oral medication is impracticable owing to persistent vomiting, the intravenous preparations of aureomycin and terramycin are probably of great value and may be life-saving in such cases.

\section{Urinary Tract}

The new antibiotics are of undoubted value in resistant urinary tract infections. They do not, however, supplant the accepted forms of therapy nor should they be used without full investigation. Alkali therapy and sulphonamides remain the, primary treatment of choice, and radical treatment of underlying structural lesions or calculi should be carried out. Infections are, however, frequently encountered, due to $B$. coli, Streptococcus faecalis, proteus or Ps. pyocyanea, which are resistant to the above treatment. In these cases sensitivity shoulæ్ be tested to streptomycin as well as to aureomycin $\frac{3}{8}$ chloramphenicol and terramycin, and the appro priate antibiotic should be used. The urine shouldbe repeatedly examined since new resistant or $\overrightarrow{\bar{\hbar}}$ ganisms may appear and require a change of antibiotic.

\section{Nervous System}

Pyogenic meningitis. All three antibiotics are of value against penicillin-resistant meningeal infec $s$ tions. Chloramphenicol most readily penetrateș into the C.S.F. and $H$. influenzae meningitis has been successfully controlled by oral administration of this drug. Aureomycin is probably the most effective treatment of staphylococcal meningitis Although in health aureomycin and terramycin do not penetrate the blood-C.S.F. barrier there्巳 is evidence that they may do so when meningition is present. Recently successful results have been obtained by intrathecal administration of aureo mycin or chloramphenicol.

Herpes zoster. Early claims of rapid cure of zoster by aureomycin have not been substantiated A controlled trial has shown that there is no shortening of the disease or prevention of postes herpetic pain by. either aureomycin or chloram $\overrightarrow{0}$ phenicol.

\section{Bacteriaemias}

Penicillin-resistant endocarditis. Any one of the new antibiotics may be of value if the organism are highly sensitive. Combined therapy, for exक्ळ ample penicillin with streptomycin, is worthy of trial in cases resistant to penicillin alone. A doub $\vec{E}$ about the value of the newer antibiotics has beep introduced by the possibility that their bacterio static activity may diminish the bactericidal action. of penicillin.

\section{Local Infections}

Ophthalmic infections. Good results have bee obtained with aureomycin and terramycin by loca application (drops or ointment) in follicular conjunctivitis, blepharitis and trachoma. Local ap응 plication may need to be supplemented by orat administration.

Burns and wound infections. All three antis biotics are of value in preventing or treating superficial infections especially those caused bP staphylococci. Successful treatment of wounds infected by Proteus vulgaris and Ps. pyocyanea has recently been reported using a solution chloramphenicol in propylene glycol.

Skin infections. Claims of successful treatment in individual patients have been made for a variet of conditions such as pyoderma, pemphigus, $\mathrm{D}_{\mathrm{B}}$ herpetiformis and furunculosis. Local staphylo $\frac{?}{\mathrm{D}}$ 
coccal lesions may sometimes clear dramatically after application of chloramphenicol or terramycin in cream or ointment form, but it is doubtful whether the use of these antibiotics is justifiable in chronic dermatitis where no specific bacterial cause can be demonstrated. The risk of sensitization and of bacterial resistance is a serious one and prolonged local application of antibiotics which may subsequently be administered orally should be avoided.

Local suppuration. The value of any antibiotic in the treatment of local abscesses, e.g. cerebral, pelvic, subphrenic or pulmonary is restricted by poor access of the drug to the bacteria in adequate concentrations. Systemic treatment may be of value in preventing spread of the infection or in combating bacteriaemia, but the walled-off infection itself can be reached only by direct instillation of antibiotic into the abscess. All three antibiotics may be used in this way in staphylococcal infections although the efficacy of such treatment is unpredictable. The use of streptococcal enzymes in closed infections such as empyema may prove of great value by rendering the organisms more accessible to the antibiotic.

It is difficult to give any comparative assessment of chloramphenicol, aureomycin and terramycin in the treatment of infections. If there is general sensitivity to all three the differences between them for systemic administration are of minor signifi- cance. As mentioned previously, chloramphenicol has a depressant effect on the bone marrow which may appear after prolonged administration; being neutral in reaction it is more appropriate for local application either as powder or in suspension. It is also more diffusible so that effective concentrations may be more easily attained in meningitis and ophthalmic infections. Aureomycin and terramycin may be given intravenously-an advantage where oral administration is impracticable, for example in the treatment of severely ill children or adults with severe vomiting or inability to swallow. The elixirs of chloramphenicol and terramycin are of special value in treating infants and young children.

In conclusion the newer antibiotics should be used with discrimination. Although they can be given by mouth there are certain disadvantages, including their expense, the risk of fungal infection and of gastro-intestinal disturbance; these should be taken into account. Antibiotic therapy as a substitute for full and accurate diagnosis is bad practice and often harmful to the patient. Penicillin is still the antibiotic of choice and when the response to this has been unsatisfactory further aid should be sought, preferably in hospital, on the reasons for this. Bacteriological diagnosis and sensitivity testing are essential for the most effective choice of the antibiotics and bacteriological control is necessary throughout the course of? treatment.

\title{
$\mathrm{HOW}$
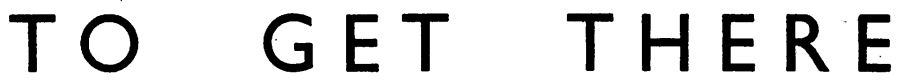

An Address Book for the Medical profession, showing how to reach the various Colleges, Societies, Institutes and Hospitals in and near London. Third edition : 1950.

\author{
Published by the \\ 60 Portland Place, London, W. I \\ Price 2s. 6d.
}

FELLOWSHIP OF POSTGRADUATE MEDICINE

\section{RUTHIN CASTLE, NORTH WALES}

A Clinic for the diagnosis and treatment of Internal Diseases (except Mental or Infectious Diseases). The Clinic is provided with a staff of doctors, technicians and nurses.

The surroundings are beautiful. The climate is mild. There is central heating throughout. The annual rainfall is $\mathbf{3 0 . 5}$ inches, that is, less than the average for England.

The Fees are inclusive and vary according to the room occupied.

For particulars apply to THE SECRETARY, Ruthin Castle, North Wales. 